\title{
Endothelial cells promote the proliferation and migration of Schwann cells
}

\author{
De-Hua Meng ${ }^{1 *}$, Jia-Peng Zou ${ }^{1 \#}$, Qin-Tong Xu ${ }^{1}$, Jia-Yi Wang ${ }^{1}$, Jie-Qin Yu ${ }^{1}$, Ya Yuan ${ }^{1}$, Zeng-Gan Chen ${ }^{1}$, \\ Ming-He Zhang ${ }^{2}$, Li-Bo Jiang ${ }^{1}$, Jian Zhang ${ }^{1}$ \\ ${ }^{1}$ Department of Orthopedics, Zhongshan Hospital, Fudan University, Shanghai, China; ${ }^{2}$ Department of Orthopedics, The 455th Hospital of Chinese \\ People's Liberation Army, Shanghai, China \\ Contributions: (I) Conception and design: DH Meng, J Zhang; (II) Administrative support: J Zhang; (III) Provision of study materials or patients: \\ DH Meng, JP Zou; (IV) Collection and assembly of data: JP Zou, QT Xu, LB Jiang; (V) Data analysis and interpretation: DH Meng, JP Zou; (VI) \\ Manuscript writing: All authors; (VII) Final approval of manuscript: All authors. \\ "These authors contributed equally to this work and should be considered as co-first authors. \\ Correspondence to: Jian Zhang; Libo Jiang. Department of Orthopedics, Zhongshan Hospital, Fudan University, 180 Fenglin Road, Shanghai, China. \\ Email: zhang.jian@zs-hospital.sh.cn; jiang.libo@zs-hospital.sh.cn.
}

Background: After peripheral nerve injury, Schwann cells proliferate and migrate to the injured site, thereby promoting peripheral nerve regeneration. The process is regulated by various factors. Endothelial cells participate in the process via angiogenesis. However, the effects of endothelial cells on Schwann cells are not yet known. The present study sought to evaluate whether endothelial cells accelerate Schwann cell proliferation and migration.

Methods: We established a co-culture model of rat Schwann cells (RSC96s) and rat aortic endothelial cells (RAOECs), and studied the effects of endothelial cells on Schwann cells by evaluating changes in Schwann cell proliferation and migration and related multiple genes and their protein expressions in the co-culture model.

Results: The results showed that increasing the proportion of endothelial cells in the co-culture model enhanced the proliferation. At days 1 and 3 following the co-culturing, the relative growth rates of the cocultured cells were $122.87 \%$ and $127.37 \%$, respectively, which showed a significant increase in the viability compared to that of the RSC96s $(\mathrm{P}<0.05)$. In this process, the expression of Ki67 increased. The migration ability of Schwann cells was also enhanced. The migration capacity of Schwann cells was detected by woundhealing and Transwell assays. The results of the group with $15 \%$ of endothelial cells was significantly higher than the results of the other groups $(\mathrm{P}<0.0001$ and $\mathrm{P}<0.05$, respectively). Further, neuregulin 1 and glial fibrillary acidic protein increased the process of Schwann cell migration.

Conclusions: The results showed that endothelial cells can promote the proliferation and migration of Schwann cells and participate in peripheral nerve regeneration.

Keywords: Peripheral nerve regeneration; cell co-culture; Schwann cells; endothelial cells

Submitted Nov 05, 2021. Accepted for publication Jan 18, 2022.

doi: 10.21037/atm-22-81

View this article at: https://dx.doi.org/10.21037/atm-22-81

^ ORCID: 0000-0001-7950-548X. 


\section{Introduction}

Schwann cells play a crucial role in the process of peripheral nerve regeneration. After peripheral nerve injury (PNI), the response of Schwann cells to injury varies. Some studies $(1,2)$ examined the dedifferentiation of Schwann cells, while others (3-6) have examined activation or reprogramming. These cells participate in the clearance of myelin and axon debris, the formation of Büngner bands, the regeneration of axons accompanied by changes in repair-related phenotypes, and the secretion of soluble factors. To accelerate regeneration after PNI, the response of Schwann cells (e.g., proliferation, migration, differentiation, and myelination) needs to be effectively regulated.

Some classic and emerging intracellular signaling pathways are involved in the proliferation and migration of Schwann cells. The cell division cycle 42 (CDC42)/ focal adhesion kinase (FAK) pathway has been proven to be required for normal Schwann cell proliferation (7). In another study (8), the allopregnanolone-mediated $\gamma$-amino butyric acid type A (GABA-A) dependent mechanism was shown to activate Src, which interacted with FAK to modulate the effect of CDC42. In that study, Schwann cell migration was also found to be determined by the same pathway. Schwann cells require Yes-associated protein 1 (YAP)/transcriptional co-activator with PDZ binding motif (TAZ) to enter the $S$-phase to proliferate, and in the absence of YAP/TAZ, there will be insufficient Schwann cells for axon sorting $(9,10)$. The extracellular-signalregulated kinase-signaling pathway (11) and mitogenactivated protein kinases (MAPKs) (12) also modulate Schwann cell proliferation.

Endothelial cells are simple flat cells that cover the inner surface of blood vessels, which are an important part of the blood vessel wall. In normal nerve tissue, blood vessels are abundant, and a network of capillaries is distributed in the intima of the nerve, ensuring the formation of nerve fibers and normal functions. Nerve regeneration can be enhanced by manipulating vascularization. After PNI, endothelial cells form micro-vessels rapidly to provide blood supply. This process is regulated by the vascular endothelial growth factor (VEGF) and plays an important role in the early regeneration of peripheral nerves $(13,14)$. Recent research (14) has examined the interaction between Schwann cells and endothelial cells. Ramos et al. found that the migration of endothelial cells can be enhanced by Schwann cells after PNI (15). Endothelial cells can also generate cytokines, such as VEGF, which participate in the regulation of Schwann cells, to speed up peripheral nerve regeneration (14,15). Previous studies mainly focused on explaining the effect of Schwann cells on endothelial cells, but few studies explored the regulatory effect of endothelial cells on Schwann cells. Peripheral nerve repair is a complex process. All cells in the local microenvironment may regulate Schwann cells. This study aims to explore the regulatory effect of endothelial cells on Schwann cells from the cellular level, so as to provide a basis for further exploring the regulation of microenvironment on Schwann cells in the process of nerve repair.

We present the following article in accordance with the MDAR reporting checklist (available at https://atm. amegroups.com/article/view/10.21037/atm-22-81/rc).

\section{Methods}

We establish a Schwall cell and endothelial cells co-cultured model to test the proliferation and migration of Schwann cells by cell proliferation assays and cell migration assays. Then we detect the related factors, Cers2, Ki67, GFAP and NRG1 to explore the potential mechanism.

\section{Cell cultures}

Rat Schwann cells (RSC96) $\left(\right.$ ATCC $^{\circledR}$ CRL-2941 $^{\text {TM }}$; Gaithersburg, MD, USA) were cultured in Dulbecco's Modified Eagle Medium (Gibco) containing $100 \mathrm{~mL} / \mathrm{L}$ of fetal bovine serum (FBS) (10099-141, Gibco; Grand Island, NY, USA), $100 \mathrm{kU} / \mathrm{L}$ of penicillin, and $100 \mathrm{mg} / \mathrm{L}$ of chloramphenicol (15140-122, Gibco) at $37{ }^{\circ} \mathrm{C}$ under humidified $5 \%$ carbon dioxide $\left(\mathrm{CO}_{2}\right)$. The RSC96s were passaged no more than 3 times with $0.25 \%$ trypsin (25300054, Gibco) before the experiments, and were observed under an inverted microscope.

Rat aortic endothelial cells (RAOECs) (BNCC337896, BeNa Culture Collection; Beijing, China) were cultured in endothelial growth medium (EGM) (Sigma-Aldrich; Darmstadt, Germany) comprising endothelial basic medium supplemented with (\%v/v) 2\% FBS, 0.04\% hydrocortisone, $0.4 \%$ human fibroblast growth factor B, $0.1 \%$ VEGF, $0.1 \%$ R3-insulin-like growth factor-I, $0.1 \%$ ascorbic acid, $0.1 \%$ human endothelial growth factor, $0.1 \%$ gentamicin/ amphotericin-B (GA-1000), and $0.1 \%$ heparin (all from Lonza EGM-2 BulletKit, Catalog \#: CC-3162; Lonza Group Ltd., Basel, Switzerland). 
Table 1 List of primer used

\begin{tabular}{llc}
\hline Gene & Direction & Sequence \\
\hline NRG1 & Forward & AGCTGGAGTAATGGGCACAC \\
& Reverse & GAGTCAGGGGTTCTCTGGC \\
GFAP & Forward & TGGAACAGCAAAACAAGGCG \\
& Reverse & TCTCCTCCTCCAGCGATTCA \\
Cers2 & Forward & ATGCTCCAGACCTTGTATGACT \\
& Reverse & CTGAGGCTTTGGCATAGACAC \\
Ki67 & Forward & ATCATTGACCGCTCCTTAGGT \\
& Reverse & GCTCGCCTTGATGGTTCCT \\
GAPDH & Forward & AAGAAGGTGGTGAAGAGG \\
& Reverse & TGTCATTGAGAGCAATGCCAGC \\
\hline
\end{tabular}

\section{Co-cultures}

To evaluate the effects of endothelial cells on Schwann cell proliferation and migration, $2 \times 10^{5}$ RSC96s were seeded into 6-well plates per well with a serum-free medium. Complete medium and the RAOECs filled the upper portion of the 0.4- $\mathrm{mm}$-pore co-culture Transwell chamber (Corning; NY, USA) in proportions of $5 \%, 10 \%$, and $15 \%$ to create a non-direct contact co-culture model. Only the complete medium for RAOEC was added to the upper chamber for the control group.

\section{Cell proliferation assays}

At days 1, 3, 5, and 7 following co-culturing with RSC96 and $5 \%$ RAOECs, a Cell Counting Kit-8 (CCK-8) (Beyotime, Nantong, China) colorimetric assay was performed in accordance with the manufacturer's instructions to assess the effects of RAOECs on Schwann cell viability and proliferation. The optical density was measured at $450 \mathrm{~nm}$ with the use of a microplate reader (Thermo Scientific; Waltham, UK).

\section{Cell migration assay}

\section{Wound-healing assay}

Schwann cells were seeded into 6 -well plates at $1 \times 10^{6}$ cells per well in the lower chamber. The upper chamber was filled with complete medium and RAOECs in the proportion of $0 \%, 5 \%, 10 \%$, and $15 \%$. When the confluence of cells was $80-90 \%$, scratches were made on the monolayer surface of cells using $200-\mu \mathrm{L}$ pipette tips. At $1 \mathrm{~h}$ before scratching, the cell medium was changed to serumfree medium, and $1 \mu \mathrm{g} / \mathrm{mL}$ of mitomycin C (CAS: 50-07-7, Sigma-Aldrich; Merck KGaA; Darmstadt, Germany) was added to each well. Subsequent to scratching, the cells were washed with serum-free medium, and grown in the medium with $10 \% \mathrm{FBS}$ in a cell incubator at $37^{\circ} \mathrm{C}$ with $5 \% \mathrm{CO}_{2}$. Images of the cells in each group were captured at $0,12,24$, 36 , and $48 \mathrm{~h}$. The relative mobility was calculated according to the gap size using the following formula: relative mobility $=1-$ gap size of the initial scratches/gap size of scratches at each time point.

\section{Transwell assays}

Cell migration was evaluated by Transwell assays using 8- $\mu$ m-pore Transwell migration chambers (Corning; NY, USA). Briefly, $2 \times 10^{5}$ RSC96s were seeded in the upper portion of a chamber with serum-free medium. The lower chambers were filled with complete medium and RAOECs with different proportions as an attractant. At 8 and $24 \mathrm{~h}$ after incubation, the non-migrated cells in the upper chamber were removed by cotton swabs. The migrated cells were stained with $0.1 \%$ crystal violet (Beyotime; Nantong, China) for $30 \mathrm{~min}$, and then photographed and counted under an optical microscope (Olympus; Tokyo, Japan).

\section{$q R T-P C R$}

At $72 \mathrm{~h}$ following co-culturing, total ribonucleic acid (RNA) was isolated using TRIzol reagent (15596-018, Invitrogen; Carlsbad, California, USA). The SYBR Premix Ex Taq (RR420A, Takara Bio; Dazin City, Japan) was used to assess neuregulin 1 (NRG1), glial fibrillary acidic protein (GFAP), ceramide synthase 2 (Cers 2$)$, and Ki67 gene expression. The reference household gene used to normalize the amount of messenger RNA in the cultures was glyceraldehyde-3phosphate-dehydrogenase (GAPDH). The sequences for the following primers were carefully examined, and their specificity was checked (see Table 1). Relative changes in gene expression were calculated using the comparative $\Delta$ crossover-threshold method.

\section{Western blot analysis}

After co-culturing for $72 \mathrm{~h}$, the Schwann cells from the bottom layer were collected. The protein concentration was determined by a bicinchoninic acid protein assay kit (Beyotime; Nantong, China) in accordance with the 

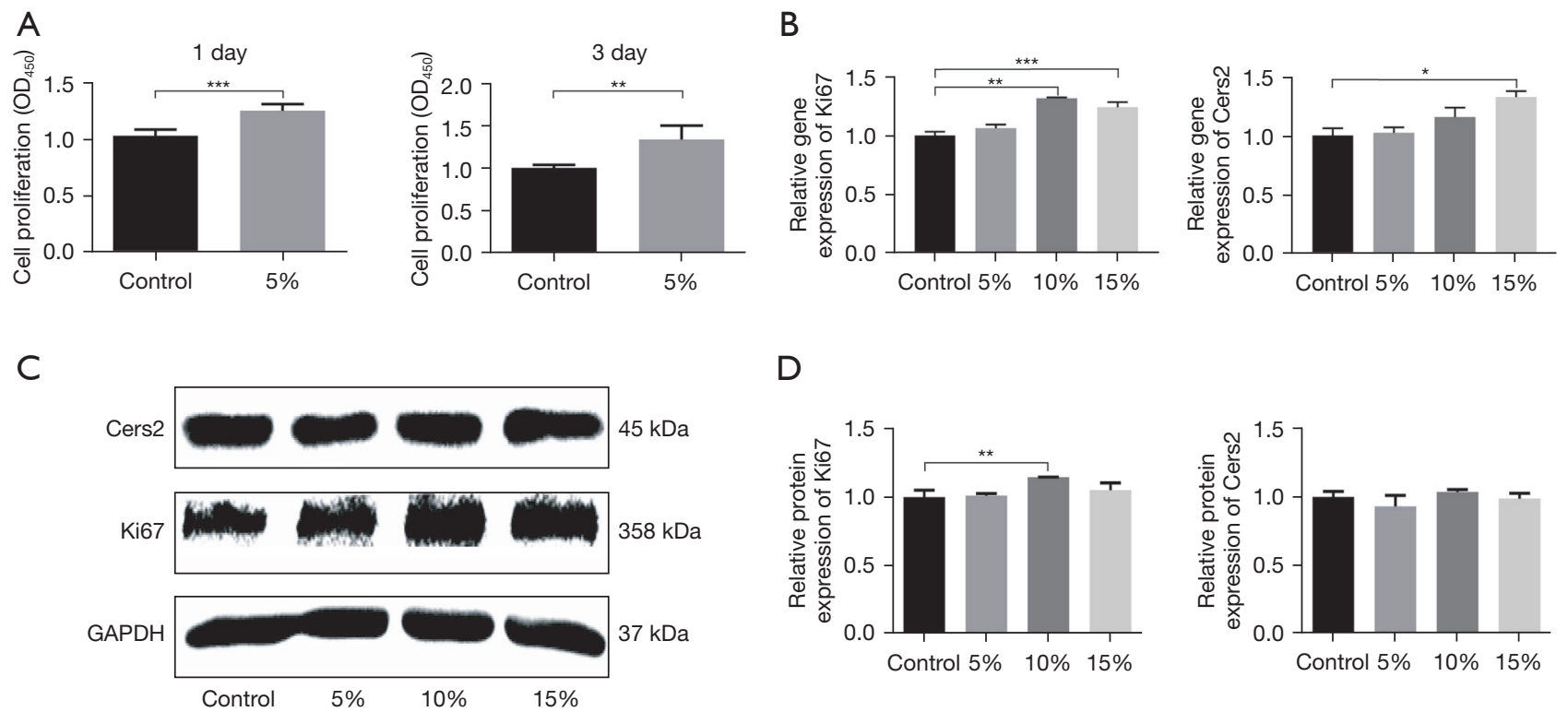

Figure 1 Endothelial cells promote the proliferation of Schwann cells. (A) At days 1 and 3 following co-culturing with 5\% RAOECs, the relative growth rates of co-culture cells increased to $122.87 \%$ and $127.37 \%$, respectively. (B) The results of the gene analysis showed that Ki67 peaked in the co-culture model with 10\% RAOECs, and the gene expression level of Cers 2 was significantly upregulated with $15 \%$ RAOECs. (C,D) The protein expression of Ki67 showed the same trend in relation to gene levels, but there was no significant difference in protein expression levels among the Cers2 groups. Mean values \pm SEM are indicated $(\mathrm{n}=3) .{ }^{*}, \mathrm{P}<0.05 ;{ }^{* *}, \mathrm{P}<0.01 ;{ }^{* * *}, \mathrm{P}<0.001$. RAOECs, rat aortic endothelial cells; Cers2, ceramide synthase 2; SEM, standard error of the mean.

manufacturer's protocol. Equal amounts of protein extracts were applied to $10 \%$ sodium dodecyl sulfate-polyacrylamide gels at $80 \mathrm{~V}$ for $20 \mathrm{~min}$, then $120 \mathrm{~V}$ for $50 \mathrm{~min}$, followed by a transfer to a polyvinylidene difluoride membrane at $15 \mathrm{~V}$ for $30 \mathrm{~min}$. The membrane was incubated with rabbit polyclonal antibody against anti-NRG1 antibody (Abcam Cat\# ab53104, RRID:AB_881750; Cambridge, UK), antiGFAP antibody (Abcam Cat\# ab7260, RRID:AB_305808; Cambridge, UK), anti-Ki67 antibody (Abcam Cat\# ab16667, RRID:AB_302459; Cambridge, UK), or mouse antibody against GAPDH (Abcam Cat\# ab8245, RRID:AB_2107448; Cambridge, UK) and anti-Cers2 antibody (Abcam Cat\# ab85567, RRID:AB_1925170; Cambridge, UK). The protein bands were exposed using a scanner.

\section{Statistical analysis}

The experiments were repeated 3 times, and the values are expressed as the mean \pm standard error of the mean (SEM). The data analysis was performed using the Student's $t$-test and a one-way analysis of variance followed by Bonferroni's multiple comparisons with GraphPad Prism 7.0 (GraphPad
Software, Inc.; San Diego, CA, USA). A P $<0.05$ indicated a statistically significant difference.

\section{Results}

\section{Endothelial cells promote the proliferation of Schwann cells}

Cell viability and proliferation were detected by CCK8 assays at days $1,3,5$, and 7 following co-culturing with RSC96s and 5\% RAOECs. At days 1 and 3 following the co-culturing, the relative growth rates of the co-cultured cells were $122.87 \%$ and $127.37 \%$, respectively, which showed a significant increase in the viability compared to that of the RSC96s $(\mathrm{P}<0.05$; see Figure $1 A)$. At days 5 and 7, due to the excessive growth of RSC96s and the huge consumption of the culture medium, the cells were in poor condition, and a large number of RSC96s died. The coculture cells survived more than the single RSC96 cultures, but the results of these 2 days were false positives, which could not provide evidence of enhanced proliferation. These results demonstrated that endothelial cells promote the proliferation of Schwann cells, and the optimal processing time was 1 or 3 days. 


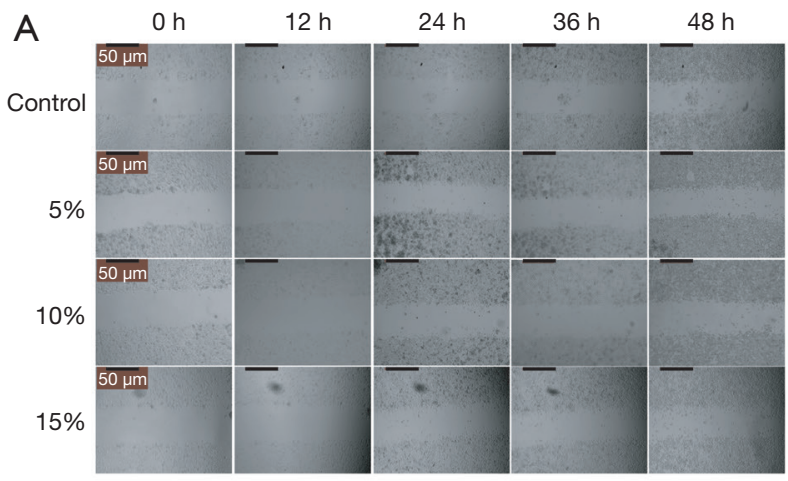

B
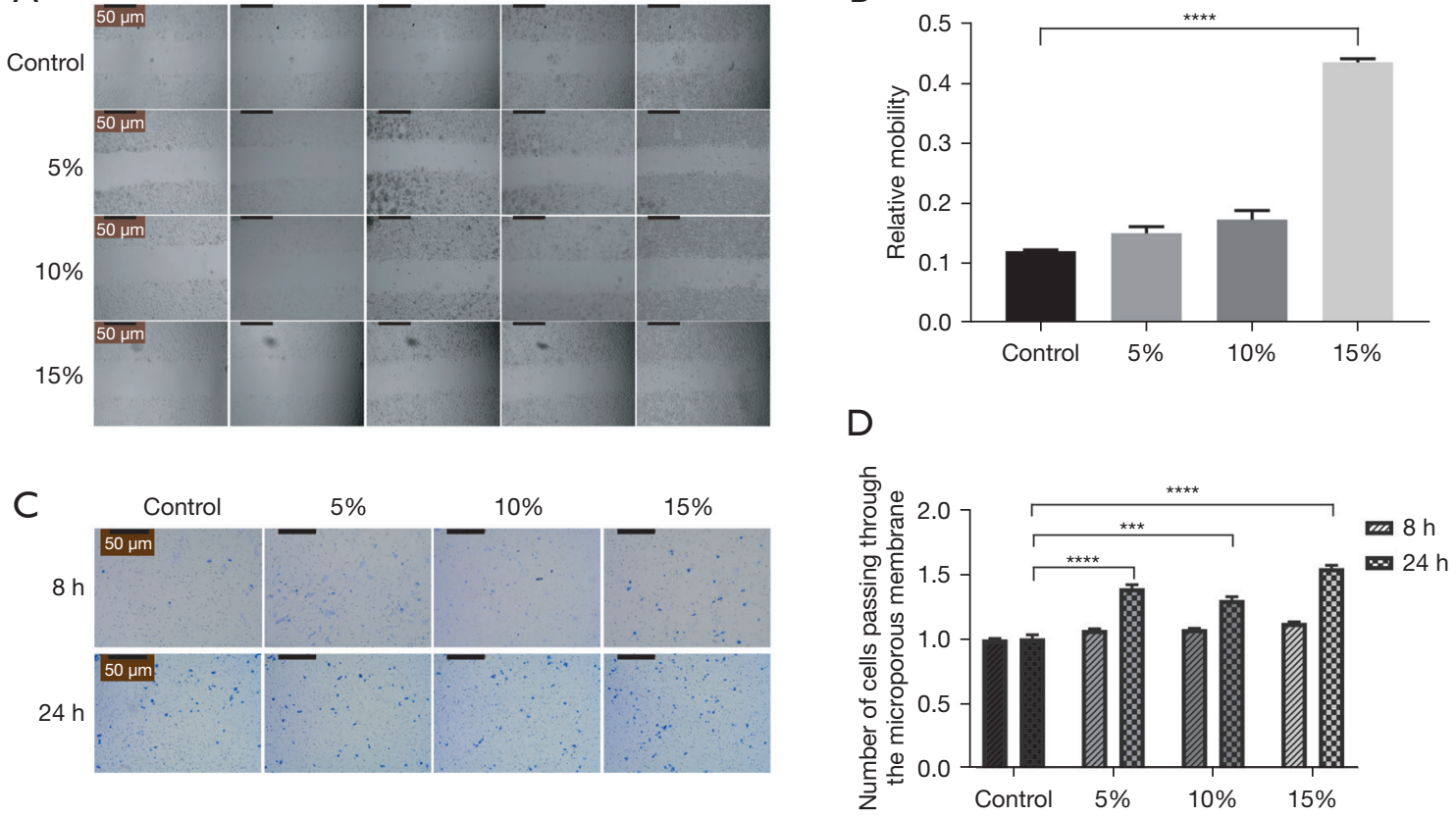

Figure 2 Endothelial cells promote the migration of Schwann cells. (A) Images of the cells in each group were captured under a light microscope $(\times 4)$. As the proportion of vascular endothelial cells increased, the area of scratches decreased. (B) Compared to the single Schwann cell group, the migration ability of Schwann cells co-cultured with $15 \%$ RAOECs was significantly enhanced. (C) For the Transwell assay, the migrated cells were stained with crystal violet at 8 and $24 \mathrm{~h}$ after incubation. (D) The relative number of cells peaked in $15 \%$ RAOECs at 8 and $24 \mathrm{~h}\left(1.13 \pm 0.01\right.$ and $1.55 \pm 0.02$, respectively). ${ }^{* *}, \mathrm{P}<0.001 ;{ }^{* * *}, \mathrm{P}<0.0001$. RAOECs, rat aortic endothelial cells.

To verify this facilitation effect on the proliferation of Schwann cells and explore the molecular mechanism, we selected 2 well-known factors (i.e., Cers2 and Ki67) for detection by quantitative reverse transcription polymerase chain reaction (qRT-PCR) and Western blot analysis. The genetic analysis results for Ki67 showed an increase in the co-culture model with proportions of $10 \%$ and $15 \%$ RAOECs. The peak of gene expression was at the proportion of $10 \%$ RAOECs, and there was a decrease in expression at the proportion of $15 \%$ RAOECs (means \pm SEM: $1.32 \pm 0.01$ vs. $1.24 \pm 0.04 ; \mathrm{P}<0.05$; see Figure $1 B$ ). The results of the Western blot analysis for Ki67 showed the same trend as that of the qRT-PCR results. The results demonstrated that compared to the control group, the expression of Ki67 protein of RSC96s with 10\% RAOECs showed a significant increase of $1.15 \pm 0.001$-fold $(\mathrm{P}<0.05$; see Figure $1 C, 1 D)$. The results of the other 2 groups also improved, but did not differ significantly to the results of the control group. For Cers2, the qRT-PCR results also showed that compared to the control group, the gene expression level of Cers 2 increased to $1.33 \pm 0.05$-fold that of the co-cultured cells with a proportion of $15 \%$ RAOECs $(\mathrm{P}<0.05)$, but the gene levels of the other 2 groups showed no significant increase to that of the control group (see Figure $1 B$ ). Inconsistent with the results of the qRT-PCR, there was a downward trend in the protein levels at the proportion of $5 \%$ RAOECs $(0.93 \pm 0.05 ; \mathrm{P}>0.05)$, and the results of the other 2 groups did not differ significantly compared to the results of the control group (see Figure $1 C, 1 D)$.

The above results demonstrate that endothelial cells promote the proliferation of Schwann cells, and the optimal processing time is 1 or 3 days.

\section{Endothelial cells promote the migratory capacity of the Schwann cells}

The migration capacity of Schwann cells was detected by wound-healing and Transwell assays. One $\mu \mathrm{g} / \mathrm{mL}$ of mitomycin $\mathrm{C}$ was added to each well $1 \mathrm{~h}$ before the wound-healing assay to inhibit cell division and eliminate the interference of cell proliferation on the results. The 

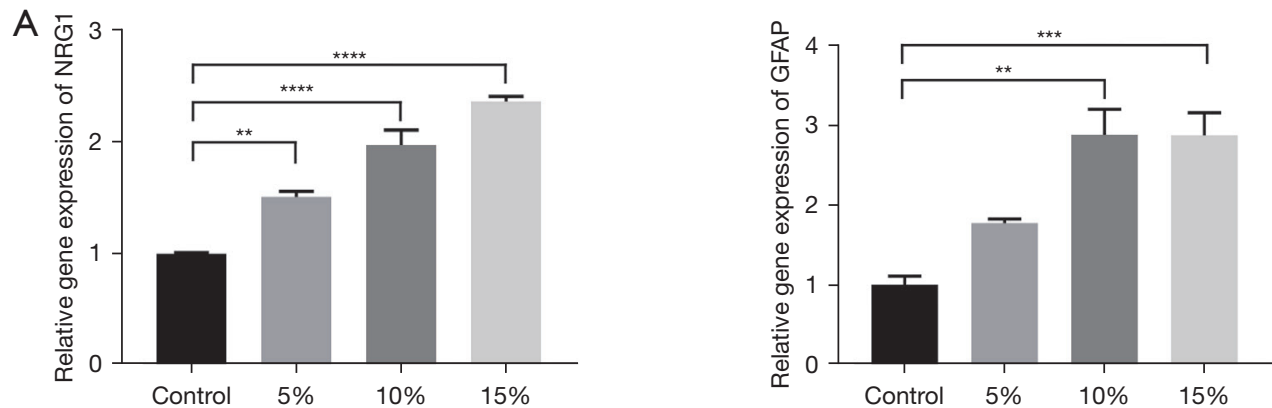

B
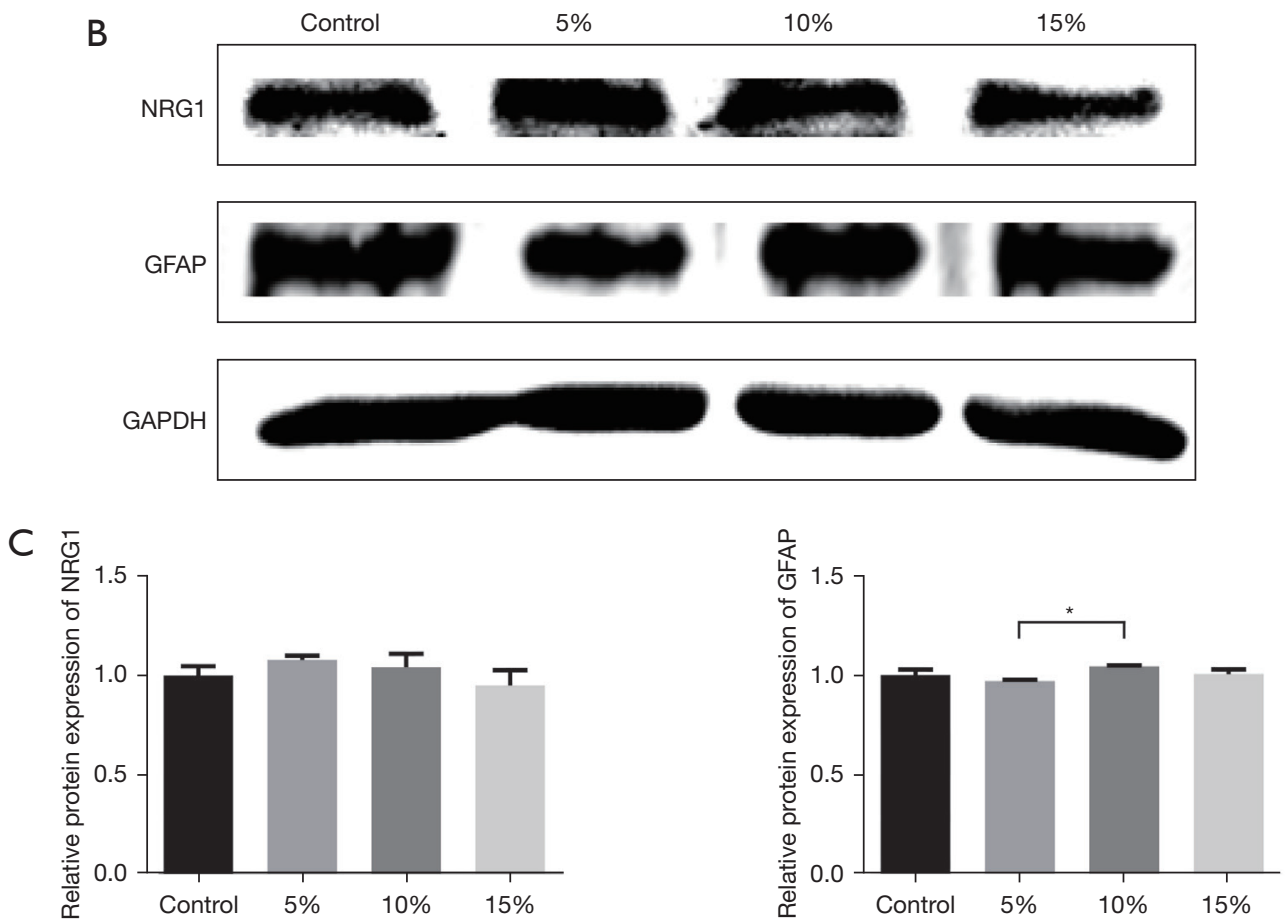

Figure 3 Endothelial cells promote the migration of Schwann cells. (A) Compared to the control group, the gene levels of NRG1 and GFAP increased significantly $(n=3)$. (B,C) Changes in the protein expression levels were not significant $(n=3)$. *,$P<0.05 ;{ }^{* *}, P<0.01 ;{ }^{* * *}, P<0.001$; ****, P<0.0001. NRG1, neuregulin 1; GFAP, glial fibrillary acidic protein; GAPDH, glyceraldehyde-3-phosphate-dehydrogenase.

results of the wound-healing assay showed that the relative migration capacities of the co-cultured cells at the proportion of $5 \%$ and $10 \%$ were $0.15 \pm 0.012$ and $0.17 \pm 0.015$, respectively, while the relative migration capacity of the $15 \%$ RAOEC group increased to $0.44 \pm 0.006$ (see Figure $2 A, 2 B$ ). Compared to the control group, the mobility of Schwann cells co-cultured with $15 \%$ of endothelial cells was significantly enhanced $(\mathrm{P}<0.0001)$. Consistent with the results of the wound-healing assay, the Transwell assay also demonstrated that migration capacity was more enhanced in the co-cultured group than the control group at both 8 and $24 \mathrm{~h}$. In addition, the results of the group with $15 \%$ RAOECs was significantly higher than the results of the other 2 groups (see Figure 2C,2D). Thus, RAOECs enhanced the migratory capacity of Schwann cells.

To further assess the effect of endothelial cells on the migration of Schwann cells, we chose 2 factors involved in the migration of Schwann cells to detect their gene and protein expression levels by qRT-PCR and Western blot analysis.

For GFAP, the gene expression level of GFAP significantly increased as the proportion of RAOECs increased. There was a $1.78 \pm 0.05$-fold increase with the proportion of $5 \%$ RAOECs $(\mathrm{P}<0.05$; see Figure $3 A)$. The other 2 groups had higher gene expression levels $(2.89 \pm 0.32$ for the $10 \%$ 
RAOECs and $2.88 \pm 0.28$ for the $15 \%$ RAOECs, respectively). There was no significant difference in the gene expression results between these 2 groups. In relation to the protein levels of GFAP, a decrease in protein expression was observed in the 5\% RAOEC group. Conversely, the results of the $10 \%$ RAOEC group was significantly increased (mean \pm SEM: $1.04 \pm 0.003$ vs. $0.97 \pm 0.004 ; \mathrm{P}<0.05$; see Figure $3 B, 3 C$ ). However, there was no significant difference between the 3 co-culture groups and the control group.

The co-culture model appeared to induce NGR1 gene expression. As the proportion of endothelial cells increased, the gene expression level of NRG1 increased significantly to $2.36 \pm 0.04$ with the $15 \%$ endothelial cell proportion $(\mathrm{P}<0.0001$; see Figure 3A). Inconsistent with the results of qRT-PCR, the results of the Western blot analysis for NRG1 showed an increase in the co-cultured model at the proportion of $5 \%$, and as the proportion increased, the protein level gradually decreased; however, these changes were not statistically significant (see Figure 3B,3C).

These results showed that endothelial cells enhance the migratory capacity of Schwann cells.

\section{Discussion}

Endothelial cells play an important role in peripheral nerve regeneration. After PNI, endothelial cells increase the blood supply of injured nerves via proliferation, migration, and angiogenesis, which promote peripheral nerve regeneration $(1,15,16)$. When PNI occurs, Schwann cells dedifferentiate and proliferate to enhance the regeneration of the axons $(1,2,13,17)$. Schwann cells also produce nerve growth factors that contribute to the survival and proliferation of axons $(1,3,11,12)$. Schwann cells have been reported to promote endothelial cell migration (15), but research on the effects of endothelial cells on the activity of Schwann cells has been limited. Thus, we hypothesized that endothelial cells regulate the activity of Schwann cells, thereby affecting the process of nerve regeneration. Previous studies have reported that VEGF promotes the proliferation and migration of Schwann cells (14), which is consistent with the hypothesis of the present study.

The present study investigated the effects of endothelial cells on the proliferation and migration of mature Schwann cells. According to Ramos et al. (15), Schwann cells can enhance the migration of endothelial cells after PNI. To eliminate the interference of endothelial cells on the results in the direct contact model, we established a nondirect contact co-culture model. The results revealed that endothelial cells promoted the proliferation of Schwann cells. The results of the gene and protein analyses for Ki67 and Cers2 were also consistent with the CCK-8 colorimetric assay, and the optimal processing time for the co-cultured cells was 1 or 3 days. We also found that the migratory capacity of Schwann cells was enhanced after co-culturing with endothelial cells, and the highest relative mobility of Schwann cells occurred in cells cocultured with $15 \%$ endothelial cells. Changes in gene and protein expressions of GFAP and NRG1 also confirmed the previous results. These results revealed that endothelial cells promote the proliferation and migration of Schwann cells.

The present study found that the proliferation of Schwann cells was enhanced when co-cultured with $5 \%$ endothelial cells. Ki67, a nuclear antigen expressed during mitosis $(18,19)$, indicates the proliferation of Schwann cells. The present study found that the gene level of Ki67 was increased after co-culturing with endothelial cells, and that the proportion of $10 \%$ had the greatest effect. The levels of protein expression were consistent with the qRT-PCR results. Peak protein expression occurred at the proportion of $10 \%$, and there was no statistical difference between the other 2 groups and the control group.

Cers2 (LASS2) is a tumor suppressor gene that inhibits cell proliferation and promotes apoptosis. It has been reported that proliferation can be enhanced by knocking out Cers 2 after sciatic nerve injury (20). Cers2, which is known as a cell proliferation inhibitor, may participate in $\mathrm{G} 0 / \mathrm{G} 1$ cell cycle arrest as previous studies $(21,22)$ described. The cell cycle arrest may via a p53-dependent pathway (23). The gene level of Cers 2 was upregulated significantly when co-cultured with $15 \%$ endothelial cells, and we found that $5 \%$ of endothelial cells was the most appropriate proportion for co-culturing with Schwann cells. No significant difference was found in the protein expression levels among the Cers 2 groups, which was inconsistent with the qRT-PCR results. We believe that this was due to the protein translation process being regulated by other factors. These results provide a new direction for the study of gene regulation in Schwann cells in peripheral nerve regeneration. These results showed that endothelial cells promote the proliferation of Schwann cells, and that $10 \%$ is the optimal proportion for the co-culturing of Schwann cells and endothelial cells to promote the proliferation of Schwann cells.

The results of the wound-healing and Transwell assays demonstrated that the co-culture promoted the migration 
of Schwann cells, of which $15 \%$ of endothelial cells had the most significant effect. GFAP is a Schwann-cellspecific cytoskeleton constituent that is upregulated after injury, and might contribute to the formation of macrocomplexes to initiate mitogenic responses and differentiate between signaling for efficient nerve regeneration (24). NRG1 (25-29) plays an important role in axonal growth, myelin formation, and re-myelin formation, promoting peripheral nerve function and regeneration. NRG1/ Erb2/3 signaling pathways may regulate the migration of Schwann cells by related molecules, including $\operatorname{Shp} 2$ which is important for the Ras/Erk1/2 pathway (30). And NRG1 may also activate the Rho-GTPase family, Cdc42 and Rac1, which are strongly associated with Schwall cells migration in vivo $(7,31,32)$. The present study showed that the gene expression levels of GFAP and NRG1 all increased after coculturing with endothelial cells, and that a proportion of $10 \%$ of endothelial cells had the greatest effect. The protein levels of co-cultured groups were increased compared to the protein levels of single Schwann cell culture. We also found that the protein levels of the other 2 groups were lower than the protein level of the group with a proportion of $10 \%$ of endothelial cells, but there was no statistically significant difference between these 2 groups and the control group. These results provide strong evidence that endothelial cells enhance the migration ability of Schwann cells.

Exosomes, known as vesicles, which carry macromolecules between cells, may mediate the regulatory process in Schwann cells. Exosomes are 30-150 nm diameter vesicles that carry mRNAs, microRNAs, and proteins, and regulate surrounding cells by paracrine. Exosomes have become the subject of increasing interest as a novel means for intercellular crosstalk. Previous reports have shown that exosomes from various cell sources contribute to the repair of PNI (33-36). According to articles by Lopez-Verrilli et al. (34) and Lopez-Leal et al. (37), exosomes derived from Schwann cells can promote axon regeneration in the peripheral nervous system. To date, few studies have been conducted on the exosomes of endothelial cells. We intend to investigate the role of exosomes in the endothelial cell promotion of Schwann cell proliferation and migration in our future research.

\section{Conclusions}

Our study found that the proliferation and migration of Schwann cells was enhanced. Consistent with our hypothesis, we also found changes in the gene and protein expression levels of 3 factors (i.e., Ki67, GFAP, and NGR1) related to the proliferation and migration of Schwann cells. The present study confirmed the effect of endothelial cells on Schwann cells, and provides novel insights into the molecular mechanisms underlying the proliferation and migration of Schwann cells.

\section{Acknowledgments}

We would like to thank Jiayun Hou for her technical assistance and support.

Funding: The study was supported by the National Science Foundation of China (No. 81870965).

\section{Footnote}

Reporting Checklist: The authors have completed the MDAR reporting checklist. Available at https://atm.amegroups. com/article/view/10.21037/atm-22-81/rc

Data Sharing Statement: Available at https://atm.amegroups. com/article/view/10.21037/atm-22-81/dss

Conflicts of Interest: All authors have completed the ICMJE uniform disclosure form (available at https://atm. amegroups.com/article/view/10.21037/atm-22-81/coif). All authors report that the research was supported by the National Science Foundation of China (No. 81870965). The authors have no other conflicts of interest to declare.

Ethical Statement: The authors are accountable for all aspects of the work in ensuring that questions related to the accuracy or integrity of any part of the work are appropriately investigated and resolved.

Open Access Statement: This is an Open Access article distributed in accordance with the Creative Commons Attribution-NonCommercial-NoDerivs 4.0 International License (CC BY-NC-ND 4.0), which permits the noncommercial replication and distribution of the article with the strict proviso that no changes or edits are made and the original work is properly cited (including links to both the formal publication through the relevant DOI and the license). See: https://creativecommons.org/licenses/by-nc-nd/4.0/.

\section{References}

1. Chen ZL, Yu WM, Strickland S. Peripheral regeneration. 
Annu Rev Neurosci 2007;30:209-33.

2. Jessen KR, Mirsky R. Negative regulation of myelination: relevance for development, injury, and demyelinating disease. Glia 2008;56:1552-65.

3. Armstrong SJ, Wiberg M, Terenghi G, et al. ECM molecules mediate both Schwann cell proliferation and activation to enhance neurite outgrowth. Tissue Eng 2007;13:2863-70.

4. Campana WM. Schwann cells: activated peripheral glia and their role in neuropathic pain. Brain Behav Immun 2007;21:522-7.

5. Webber C, Zochodne D. The nerve regenerative microenvironment: early behavior and partnership of axons and Schwann cells. Exp Neurol 2010;223:51-9.

6. Allodi I, Udina E, Navarro X. Specificity of peripheral nerve regeneration: interactions at the axon level. Prog Neurobiol 2012;98:16-37.

7. Benninger Y, Thurnherr T, Pereira JA, et al. Essential and distinct roles for cdc42 and rac1 in the regulation of Schwann cell biology during peripheral nervous system development. J Cell Biol 2007;177:1051-61.

8. Melfi S, Montt Guevara MM, Bonalume V, et al. Src and phospho-FAK kinases are activated by allopregnanolone promoting Schwann cell motility, morphology and myelination. J Neurochem 2017;141:165-78.

9. Grove M, Kim H, Santerre M, et al. YAP/TAZ initiate and maintain Schwann cell myelination. Elife 2017;6:20982.

10. Poitelon Y, Lopez-Anido C, Catignas K, et al. YAP and TAZ control peripheral myelination and the expression of laminin receptors in Schwann cells. Nat Neurosci 2016;19:879-87.

11. Napoli I, Noon LA, Ribeiro S, et al. A central role for the ERK-signaling pathway in controlling Schwann cell plasticity and peripheral nerve regeneration in vivo. Neuron 2012;73:729-42.

12. Kim HA, Mindos T, Parkinson DB. Plastic fantastic: Schwann cells and repair of the peripheral nervous system. Stem Cells Transl Med 2013;2:553-7.

13. Jessen KR, Mirsky R. The repair Schwann cell and its function in regenerating nerves. J Physiol 2016;594:3521-31.

14. Hobson MI, Green CJ, Terenghi G. VEGF enhances intraneural angiogenesis and improves nerve regeneration after axotomy. J Anat 2000;197 Pt 4:591-605.

15. Ramos T, Ahmed M, Wieringa P, et al. Schwann cells promote endothelial cell migration. Cell Adh Migr 2015;9:441-51.

16. Storkebaum E, Lambrechts D, Carmeliet P. VEGF: once regarded as a specific angiogenic factor, now implicated in neuroprotection. Bioessays 2004;26:943-54.

17. Jessen KR, Mirsky R, Lloyd AC. Schwann Cells: Development and Role in Nerve Repair. Cold Spring Harb Perspect Biol 2015;7:a020487.

18. Falini B, Flenghi L, Fagioli M, et al. Evolutionary conservation in various mammalian species of the human proliferation-associated epitope recognized by the Ki-67 monoclonal antibody. J Histochem Cytochem 1989;37:1471-8.

19. Ezhkova E, Pasolli HA, Parker JS, et al. Ezh2 orchestrates gene expression for the stepwise differentiation of tissuespecific stem cells. Cell 2009;136:1122-35.

20. Yu B, Zhou S, Wang Y, et al. miR-221 and miR-222 promote Schwann cell proliferation and migration by targeting LASS2 after sciatic nerve injury. J Cell Sci 2012;125:2675-83.

21. Zeng F, Huang L, Cheng X, et al. Overexpression of LASS2 inhibits proliferation and causes G0/G1 cell cycle arrest in papillary thyroid cancer. Cancer Cell Int 2018;18:151.

22. Su J, Yu W, Gong M, et al. Overexpression of a Novel Tumor Metastasis Suppressor Gene TMSG1/LASS2 Induces Apoptosis via a Caspase-dependent Mitochondrial Pathway. J Cell Biochem 2015;116:1310-7.

23. Wang Y, Qiu C, Lu N, et al. FOXD1 is targeted by miR$30 a-5 p$ and miR-200a-5p and suppresses the proliferation of human ovarian carcinoma cells by promoting p2 1 expression in a p53-independent manner. Int J Oncol 2018;52:2130-42.

24. Triolo D, Dina G, Lorenzetti I, et al. Loss of glial fibrillary acidic protein (GFAP) impairs Schwann cell proliferation and delays nerve regeneration after damage. J Cell Sci 2006;119:3981-93.

25. Gambarotta G, Fregnan F, Gnavi S, et al. Neuregulin 1 role in Schwann cell regulation and potential applications to promote peripheral nerve regeneration. Int Rev Neurobiol 2013;108:223-56.

26. Ieguchi K, Fujita M, Ma Z, et al. Direct binding of the EGF-like domain of neuregulin-1 to integrins (\{alpha\} $\mathrm{v}\{$ beta\} 3 and \{alpha\}6\{beta\}4) is involved in neuregulin-1/ ErbB signaling. J Biol Chem 2010;285:31388-98.

27. Newbern J, Birchmeier C. Nrg1/ErbB signaling networks in Schwann cell development and myelination. Semin Cell Dev Biol 2010;21:922-8.

28. Perlin JR, Lush ME, Stephens WZ, et al. Neuronal Neuregulin 1 type III directs Schwann cell migration. Development 2011;138:4639-48.

29. Stassart RM, Fledrich R, Velanac V, et al. A role for 
Schwann cell-derived neuregulin-1 in remyelination. Nat Neurosci 2013;16:48-54.

30. Mohi MG, Neel BG. The role of Shp2 (PTPN11) in cancer. Curr Opin Genet Dev 2007;17:23-30.

31. Yamauchi J, Miyamoto Y, Chan JR, et al. ErbB2 directly activates the exchange factor Dock7 to promote Schwann cell migration. J Cell Biol 2008;181:351-65.

32. Nodari A, Zambroni D, Quattrini A, et al. Beta1 integrin activates Rac1 in Schwann cells to generate radial lamellae during axonal sorting and myelination. J Cell Biol 2007;177:1063-75.

33. Bucan V, Vaslaitis D, Peck CT, et al. Effect of Exosomes from Rat Adipose-Derived Mesenchymal Stem Cells on Neurite Outgrowth and Sciatic Nerve Regeneration After Crush Injury. Mol Neurobiol 2019;56:1812-24.

34. Lopez-Verrilli MA, Picou F, Court FA. Schwann cell-

Cite this article as: Meng DH, Zou JP, Xu QT, Wang JY, Yu JQ, Yuan Y, Chen ZG, Zhang MH, Jiang LB, Zhang J. Endothelial cells promote the proliferation and migration of Schwann cells. Ann Transl Med 2022;10(2):78. doi: 10.21037/ atm-22-81 derived exosomes enhance axonal regeneration in the peripheral nervous system. Glia 2013;61:1795-806.

35. Mead B, Tomarev S. Bone Marrow-Derived Mesenchymal Stem Cells-Derived Exosomes Promote Survival of Retinal Ganglion Cells Through miRNA-Dependent Mechanisms. Stem Cells Transl Med 2017;6:1273-85.

36. Shi Q, Qian Z, Liu D, et al. GMSC-Derived Exosomes Combined with a Chitosan/Silk Hydrogel Sponge Accelerates Wound Healing in a Diabetic Rat Skin Defect Model. Front Physiol 2017;8:904.

37. Lopez-Leal R, Court FA. Schwann Cell Exosomes Mediate Neuron-Glia Communication and Enhance Axonal Regeneration. Cell Mol Neurobiol 2016;36:429-36.

(English Language Editor: L. Huleatt) 